\title{
Criminologie
}

\section{La réforme pénale et la réciprocité des droits}

\section{Alvaro P. Pires}

Volume 24, numéro 1, 1991

Les droits des détenu-e-s

URI : https://id.erudit.org/iderudit/017304ar

DOI : https://doi.org/10.7202/017304ar

Aller au sommaire du numéro

Éditeur(s)

Les Presses de l'Université de Montréal

ISSN

0316-0041 (imprimé)

1492-1367 (numérique)

Découvrir la revue

Citer cet article

Pires, A. P. (1991). La réforme pénale et la réciprocité des droits. Criminologie, 24(1), 77-104. https://doi.org/10.7202/017304ar

\section{Résumé de l'article}

This letter deals with two major questions. The first shows how the "program" of criminology which emerged in the 19th century contributes to a neutralisation of the impact of discussions concerning human rights in the case of punishment. To do this, the author compares two paradigms of penal reform. The second question tries to clarify the relations established in Quebec between the rights of prisoners and abolitionist alternatives from 1975 on.
Ce document est protégé par la loi sur le droit d'auteur. L'utilisation des services d’Érudit (y compris la reproduction) est assujettie à sa politique d'utilisation que vous pouvez consulter en ligne.

https://apropos.erudit.org/fr/usagers/politique-dutilisation/ 
This letter deals with two major questions. The first shows how the "program» of criminology which emerged in the 19th century contributes to a neutralisation of the impact of discussions concerning human rights in the case of punishment. To do this, the author compares two paradigms of penal reform. The second question tries to clarify the relations established in Quebec between the rights of prisoners and abolitionist alternatives from 1975 on.

Le mot «incarcérées», dans l'expression «droits des personnes incarcérées», est souvent trompeur, car trop restrictif, lorsqu'il est employé pour caractériser les mouvements sociaux et groupes qui se sont occupés de cette question. En effet, ils ont aussi souvent revendiqué des droits pour les personnes accusées et en détention avant jugement (exigeant la réduction du recours à la détention, etc.), aussi bien que pour les personnes ayant purgé leur peine (victimes de discrimination en raison d'un casier judiciaire, etc.). Ces mouvements se sont aussi engagés contre l'emprisonnement à défaut de paiement d'amende et en faveur d'une série de réformes qui n'étaient pas exclusivement rattachées à la vie interne de la prison (révision des règles en matière de libération conditionnelle, etc.). Ces groupes se sont donc occupés des limites du droit de punir.

En outre, il faut attirer l'attention sur le double sens du terme «droit». En effet, ces mouvements ont revendiqué des réformes relevant de deux catégories de droits: des droits-résistances (droit de...) et des droits-créances (droit à.... $)^{1}$. En gros, les droits-résistances visent à garantir une zone d'autonomie individuelle: droit de vivre, droit de circuler, droit de vote, droit de s'exprimer, droit de choisir et de pratiquer sa religion, droit d'être protégé contre l'emprisonnement arbitraire, les châtiments cruels, etc. D'une part, ces droits-résistances limitent l'ingérence de l'État (et des particuliers) dans la vie privée des individus et, d'autre part, ils assurent des moyens d'action aux individus. Au Canada, par exemple, on a accordé beaucoup d'attention aux tribunaux disciplinaires en prison (Dumont et Landreville, 1973; Jackson,

* Directeur, Département de criminologie, Université d'Ottawa, Pavillon Tabaret, Ottawa (Ontario) KIN 6N5.

1. Sur cette distinction, voir Mourgeon (1978), Loschak (1984), Pires et Vallières (1986, pp. 80-82). 
1974), à l'usage abusif de l'isolement cellulaire (Jackson, 1983; Hattem, 1984), etc.

Les droits-créances visent surtout à promouvoir le mieux-être social des groupes défavorisés. Ils relèvent donc des services: droit à l'éducation, droit au travail rémunéré, droit aux services médicaux, droit à l'information, droit à une bonne alimentation, etc. Or, lorsqu'on pense aux mouvements des droits des personnes incarcérées, on oublie souvent ces «droits aux services», et ce malgré leur importance pour ces mouvements.

Enfin, lorsqu'on traite de cette question, il convient aussi de ne pas oublier qu'il y a eu plusieurs groupes et personnes concemés par ces droits, et par la réforme pénale en général, et qu'il ne s'agit pas ici d'un ensemble homogène: ces groupes et personnes n'ont pas nécessairement mis en valeur les mêmes (types de) droits, n'ont pas adopté les mêmes positions théoriques ou les mêmes stratégies d'action, ni n'ont affiché, le cas échéant, les mêmes couleurs politiques sur la société en général. Il y a aussi une différence, parfois difficile à cerner, entre les groupes formés (ou très proches) de personnes ayant eu des démêlés avec la justice et les groupes ou personnes cuvrant de manière plus détachée. Qui plus est, la portée et le contenu des revendications des différents mouvements ont varié énormément dans le temps et dans l'espace. Il est donc très difficile, voire périlleux, de faire une évaluation globale de ces mouvements, ou même d'un seul de ces mouvements, sans tenir compte de toutes ces dimensions et du contexte historique où se sont insérées leurs revendications.

Ma démarche ne contribuera pas beaucoup à résoudre ces difficultés. Je n'ai pas l'intention de répondre à des questions telles: «Que doit-on entendre par droits des personnes incarcérées?», «Depuis quand existe-t-il des personnes préoccupées par ces droits?», «Ces mouvements ont-ils apporté une contribution valable à la justice pénale?», «Peuvent-ils résoudre les problèmes posés par la prison?», etc. Je ne peux pas non plus répondre à la question: «La prison est-elle encore une institution "totale" (au sens de Goffman), ou est-elle devenue une autre sorte d'institution et comment la désigner ("ouverte", démocratique, etc.)?» Car la réponse à cette question appelle des développements importants, à commencer par un examen de la notion même d' «institution totale». Il faut cependant se rappeler que pour Goffman (1961, pp. 46-47), il y a cinq groupes différents d'institutions «totales» et que même les organismes qui prennent en charge les personnes inoffensives et jugées incapables de subvenir à leurs besoins (par exemple les foyers pour vieillards) font partie de ce type d'institutions. Si l'on suit Goffman il me semble donc qu'il y a très peu de chances que l'on puisse dire, sans se méprendre, que la prison n'est plus une institution totale, même s'il y a 
différents types de prisons et même si elles ont subi des changements au fil des années. Aussi ne me semble-t-il pas possible d'établir un lien entre la notion d'institution totale et les changements observés dans les prisons.

Les développements qui suivent vont, au contraire, s'articuler autour de deux questions majeures. La première vise à montrer comment le programme de réforme criminologique proposé au XIX ${ }^{e}$ siècle contribue à amortir, voire à neutraliser l'impact du discours sur les droits de la personne lorsqu'il s'agit de «justiciables ${ }^{2}$ ». Elle a une portée théorique générale et nous aidera à mieux comprendre la raison d'être de la question des droits des justiciables et ses rapports avec les orientations en matière de réforme du droit criminel. J'attirerai ici l'attention sur l'existence de deux «paradigmes» opposés de réforme pénale dès le $\mathrm{XIX}^{\mathrm{e}}$ siècle.

La deuxième question vise à élucider les rapports qui se sont établis, au Québec, entre les droits des personnes incarcérées et les «options abolitionnistes» à partir du milieu des années 1970. On sera alors aussi en mesure d'évaluer la portée et les limites d'une critique qui fut adressée à ceux qui se sont occupés de ces questions, à savoir qu'ils ne s'intéressaient pas vraiment au sort des personnes incarcérées. La question ici est la suivante: Pourquoi certains auteurs et praticiens en sont-ils venus, au Québec, à préconiser l'«abolition» plutôt que l'amélioration des prisons et quelle signification doit-on donner à leurs prises de position? Je présenterai d'abord quelques remarques générales sur l'évolution de la problématique des droits des personnes incarcérées au Québec, puis j'introduirai quelques éléments relatifs au contexte des prises de position à ce moment. Il est entendu que ma réponse à la question posée ci-dessus ne sera pas exhaustive.

En conclusion, je proposerai une réflexion sur les limites des divers «humanismes» des théories de la peine, puisque cette question est récurrente, de manière explicite ou implicite, dans le débat sur la prison et sur la réforme pénale.

\section{LE PROGRAMME DE LA CRIMINOLOGIE POSITIVISTE ET LE SILENCE SUR LES DROITS}

Je me propose d'explorer ici deux questions étroitement reliées: Comment peut-on expliquer le fait que la question des droits des personnes

2. À défaut d'une désignation plus appropriée, le terme «justiciable» désigne ici toute personne ayant eu des problèmes avec la justice pénale. Tous les termes actuellement employés pour désigner ces personnes (criminels, contrevenants, etc.) soulèvent à mon avis un certain nombre de problèmes. 
incarcérées n'ait pas été discutée de façon soutenue au XIX ${ }^{e}$ siècle alors que ces droits étaient radicalement bafoués et qu'on proclamait en même temps, dans d'autres domaines, les vertus des droits de la personne? Quels sont les rapports entre, d'une part, les objectifs que nous accordons au droit (criminel) et, d'autre part, notre conception des droits des justiciables, des victimes et de la société, et nos orientations en matière de réforme pénale en général?

D'abord, je veux attirer l'attention sur le formidable effet de «colonisation» que les théories de la peine (de la rétribution, de la dissuasion, de la réadaptation, de la neutralisation) ont exercé sur notre façon de concevoir les sanctions et les orientations de «politique juridique» en matière pénale. Mon hypothèse de travail est que toutes les théories de la peine ont contribué à renforcer la dimension stigmatisante véhiculée par le droit criminel et à légitimer un usage abusif de la prison. Ensuite, je veux dégager le «réseau» des perceptions et (l'ordre) des valeurs qui furent créées par l'acceptation du programme criminologique positiviste au $\mathrm{XIX}^{\mathrm{e}}$ siècle $^{3}$, réseau dont nous ne sommes pas encore entièrement sortis. On sait que la criminologie s'est développée en partie par opposition à la pensée classique du siècle des Lumières. Je mettrai l'accent sur la nouvelle image des justiciables et sur la priorité donnée à cet objectif de guerre à la criminalité.

La pensée classique affirmait, sous l'influence du christianisme, de l'humanisme et de la théorie libérale, que toutes les personnes étaient égales, libres et rationnelles. Rien ne distinguait fondamentalement le justiciable de sa contrepartie, l'individu respectueux des lois. La différence de conduite ne révélait rien au-delà d'elle-même: «in classical jurisprudence, the only difference between criminal and non-criminal is a contingent event: one has chosen, on occasion, to behave in a criminal fashion, whereas the other has not» (Garland, 1986, p. 90). Certes, la conception classique pouvait aussi accorder une dimension stigmatisante à la peine, comme fut le cas de la théorie rétributiviste de Kant (Pires, 1990a). Mais l'image qu'elle se faisait de la personne condamnée n'était pas fondamentalement différente de celle de la personne respectueuse des lois. Le programme criminologique va ajouter à cette tendance à imposer une sanction pénale stigmatisante une tout autre vision du justiciable. Celui-ci sera vu et présenté comme un être différent des personnes respectueuses de la loi. Le justiciable est alors conçu comme biologiquement, psychologiquement ou socialement anormal, une véritable species generis humani. Il est, par définition, un être «moralement pathologique». Le constat d'anormalité ressort automatiquement de la conduite illégale. Mais, pour ces réformateurs, il est possible de découvrir le «criminel» avant son

3. Nous renvoyons le lecteur à l'excellente analyse de Garland (1986, pp. 73-111). 
acte. Car l'acte n'est qu'un symptôme de son «état criminel». Garofalo (1914, p. 226) exprime joliment ce postulat lorsqu'il affirme:

Suppose, for example, that a criminal breach of trust is committed by a man who had hitherto followed an honest calling and been in the receipt of an adequate income. Nothing in his previous conduct, let us say, or in his conditions of life, appears in any way to be capable of impelling him to crime. Yet, it will not do to say, because of this fact, that we are here dealing with a normal man. Hardly anything could be more inaccurate, in my opinion, than the adage: "Occasion makes the thief." To be true, the phrase should be: "Occasion enables the thief to steal.»

Or, ce nouveau statut ontologique des justiciables contribue à conserver un régime et à créer un espace juridiques particuliers pour ce groupe de personnes sans que cela heurte la conscience éthique collective à un moment où le droit en général affirmait les nouvelles valeurs démocratiques et proclamait à voix haute les nouveaux droits de la personne. En effet, cette discrimination à l'égard des justiciables apparaissait alors comme légitime et entièrement justifiée par la nature des choses. La criminologie a donc contribué à conserver une série de règles juridiques d'exclusion (qui remontait à certaines expériences anciennes du droit) qui disqualifie ces personnes comme sujets de droits, et ce à un moment où on consolidait les conquêtes démocratiques. Comme cette ouverture démocratique soudaine de l'ensemble de la structure sociale au citoyen ordinaire (accès à la participation politique, à la fonction publique, au service militaire, au jury, etc.) allait à l'encontre des règles qui gouvernaient la société médiévale, elle était perçue comme à la fois attirante et menaçante (Pires, 1983, pp. 45-56). On a limité alors cette ouverture en introduisant d'innombrables exceptions à la règle du «égal pour tous» et en cherchant à mieux les justifier (Pires, 1983, p. 321).

D'un autre côté, comme l'a souligné Garland (1986, p. 103), le programme criminologique permettait de créer et de justifier l'adoption d'une forme juridiquement molle, c'est-à-dire sans limites strictes, de régulation sociale à l'égard des justiciables. Au lieu de la simple prohibition de certains actes, le programme criminologique demandait et justifiait une inspection et un contrôle des individus eux-mêmes (ibid.). La prison va constituer le lieu par excellence où s'exercera cette forme molle de régulation sociale. Mais attention: le criminologue n'est pas pour autant un loup-garou qui erre dans les prisons en quête de leur chair humaine! Beaucoup de philanthropes sont vraiment des humanistes. Mais leur humanisme fait silence sur les droits. Même à l'époque où les témoins oculaires nous abreuvent de descriptions des prisons propres à nous faire dresser les cheveux sur la tête, l'humanisme n'en demeure pas moins présent. C'est d'ailleurs en partie lui qui a motivé 
ces descriptions. Mais Laplante (1989, pp. 111-121) nous montre bien ce qu'il advient des bonnes intentions et des humanismes lorsqu'ils accordent la priorité à la mission de «combattre le criminel» conçu, en plus, comme un ennemi et un être anormal.

On trouve un exemple flagrant de cet «humanisme sans droits» dans ces débats pénologiques sur les grands systèmes pénitentiaires (auburnien et pennsylvanien) qui ont passionné les réformateurs pendant la première moitié du $\mathrm{XIX}^{\mathbf{e}}$ siècle. Si l'on se donne la peine de parcourir ce débat, on voit bien comment les réformateurs sont surtout absorbés et intéressés par une question d'efficacité : comment réduire le taux de criminalité et de récidive? Il est surprenant de constater le silence sur les droits alors même qu'on tente toutes sortes d'expérimentations sur des personnes. Et ce n'est pas un simple problème de «mentalité de l'époque», car au même moment, hors de cette chasse-gardée des réformateurs, on affirme les valeurs démocratiques et on discute beaucoup des droits. S'il est un trait caractéristique de cette «mentalité de l'époque», c'est bien le refus d'étendre les droits de la personne aux justiciables. Il est quand même remarquable de constater à cet égard que même l'auteur de $D e$ la démocratie en Amérique (1835-1840) n'a pas engagé une lutte pour la défense des droits des personnes incarcérées lorsqu'il a écrit, avec Beaumont, le Système pénitentiaire (1833-1845). Bien au contraire, il a justifié une prison répressive, dissuasive, rigoureuse et «sans droits» à proprement parler ${ }^{4}$. Et pourtant, il s'est bien rendu compte de la sévérité et du «spectacle du plus complet despotisme» (Beaumont et Tocqueville, 1845, p. 196) offert par les prisons américaines (voir aussi Perrot, 1984, p. 43).

En fait, le programme criminologique émergeant va contribuer fortement à atténuer, voire à effacer la ligne de démarcation entre «pénalité» et «pouvoir arbitraire», abus de pouvoir. Les adeptes de ce programme vont d'ailleurs éviter (jusqu'à la fin des années 1960) d'entamer une discussion systématique sur les droits, et ce même si on assiste à quelques changements notables dès la fin du $\mathrm{XIX}^{\mathrm{e}}$ siècle. La criminologie a donc contribué à la création d'un véritable no man's land ou presque, en matière d'exercice et de formes du pouvoir de punir. C'est cela qui a permis le développement de ce que Garland (1986, p. 90) a appelé un «correctionnalisme», un «interventionnisme» et un «étatisme» poussés à l'outrance. Ainsi, pour Garofalo, comme pour les autres représentants du programme criminologique, «la forme logique de la réaction contre le crime» (Garofalo, 1914, pp. 219-220) est l'élimination ou l'exclusion: "herein the state will be simply following the example of nature». Et il y a quatre principales formes d'exclusion ou d'élimination: la peine de mort, l'emprisonnement (en vue de réadapter), le bannissement ou la transportation

4. Sur la pensée pénologique de Tocqueville, voir l'excellent article de Perrot (1984). 
et l'expulsion de la personne de sa situation sociale particulière (par exemple en le radiant de sa profession).

Dans ce contexte, les mouvements de défense des droits des justiciables doivent être vus d'abord et avant tout comme une forme de réaction contre cette tendance à disqualifier les personnes ayant eu des démêlés avec la justice pénale, à délaisser la question des limites du droit de punir et à gommer les lignes de démarcation entre les formes de pénalité acceptables et le pouvoir arbitraire, entre les types de pénalité recevables et non recevables, etc.

Mais les mouvements pour les personnes incarcérées ont eu aussi à réagir, si l'on peut dire, contre une autre idéologie plus spécifique: celle des théories de la peine 5 . Je ne retiens pour l'instant qu'un seul point qui est directement relié à mes propos, en l'occurrence la revendication des «services» (droit à...) pour les personnes incarcérées. Logiquement parlant, c'est la théorie de la dissuasion qui fait obstacle ici, particulièrement dans la version soutenue par Bentham (1791). En effet, c'est à cette théorie que l'on doit la tristement fameuse "règle de la sévérité» (principle of the less eligibility) qui devrait gouverner les conditions de vie dans le pénitencier. Devenue leitmotiv de la pénologie carcérale, cette règle statuait, entre autres choses, que l'on ne devait pas rendre la condition des personnes incarcérées meilleure que celle des individus de la classe la plus pauvre dans la société (Bentham, 1791, pp. 20-21). Car la maison de pénitence qui ne répondrait pas à cette condition «n'aurait pas ce caractère de peine qui doit effrayer celui qui est tenté de commettre un crime». Selon la représentation imaginaire que le criminologue avait alors des pauvres et des pouvoirs magiques de la peine, si la prison n'était pas affreuse, tous allaient se précipiter pour commettre des crimes et sacrifier volontiers leur liberté afín de jouir du «confort enviable» de la prison. Parler d'améliorer les services et les conditions de vie pour les personnes incarcérées n'allait donc pas de soi : on se heurtait, et l'on se heurte encore, entre autres choses, à cette représentation de la "prison-bonbonnière ${ }^{6}$ ". Les mouvements pour les personnes incarcérées ont dû rappeler doucement au début que si l'on met un animal en cage, on est quand même plus responsable de sa vie que si l'on le laisse mourir dans la nature (Landreville, Gagnon, Desrosiers, 1976, pp. 117-118). Cependant, le plus embarrassant est que plusieurs représentants de la théorie de la réadaptation ont aussi accepté, à différents degrés, ce critère de la théorie de la dissuasion. En effet, ce que l'on trouve, le plus souvent, c'est un amalgame confus de l'idée d'intimidation avec celle de réadaptation (Laplante, 1989, p. 114).

5. J'ai traité en partie de cette question ailleurs (Pires, 1990a; 1990b).

6. L'expression «bonbonnière» est peut-être de Tocqueville (Perrot, 1984, p. 21). 
Nous allons maintenant examiner deux «paradigmes» concurrents de politique juridique au $\mathrm{XIX}^{\mathrm{e}}$ siècle. Je qualifierai le premier de «paradigme de l'éthique compréhensive» (full ethics) ou "de la réciprocité des droits» et le deuxième, de "paradigme de l'éthique totale» (total ethics) ou «de la guerre contre la criminalité» (Pires, 1990b).

\subsection{LE PARADIGME «DE L'ÉTHIQUE COMPRÉHENSIVE» (OU DE LA RÉCIPROCITÉ DES DROITS)}

J'illustrerai le premier paradigme à partir d'un essai de Spencer (1860) intitulée «Prison-Ethics». Certains de ses arguments sont aujourd'hui anachroniques et on doit le lire en conservant à l'esprit son contexte. Les thèses soutenues dans cet essai ne seront pas retenues par le programme criminologique positiviste. Pour emprunter l'expression d'Edgar Morin, il s'agit donc d'un véritable «paradigme perdu».

Le titre est significatif, puisqu'il désigne les prisons comme point d'ancrage pour une réflexion sur la question des droits. Mais il est aussi trompeur, puisqu'il porte plutôt sur la réforme pénale que sur la prison. En effet, l'objectif de Spencer (1860, p. 187) est d'établir un «code of prison-ethics» capable de servir de guide à la réforme pénale dans son ensemble. À ses yeux, il s'agit beaucoup plus d'une question d'éthique que de droits. Son point de départ est qu'il convient de déterminer, dans l'évaluation d'une situation, $\grave{a}$ la fois ce qui est «relatively right», compte tenu des contraintes du moment, et ce qui est «absolutely right»: «That is to say, though we must ever aim to do what is best to the present times, yet we must ever bear in mind what is abstractedly best; so that the changes we make be towards it, and not away from it» (p. 153). ...«the ethics of immediate experience must be enlightened by abstract ethics, to ensure correct guidance» (p. 157). La règle structurant ce paradigme est donc claire: il faut faire le mieux possible dans une conjoncture donnée, sans perdre de vue ce qui serait l'idéal à réaliser. En d'autres mots, il faut se garder de la tentation de décrire ce que nous jugeons la meilleure solution possible compte tenu des circonstances comme si c'était la solution idéale. Et inversement, si l'on rêve exclusivement à l'idéal, on a peu de chances d'infléchir les pratiques. Spencer se propose alors d'étudier la justice pénale à la lumière de ce principe général.

11 pose ensuite les questions fondamentales suivantes: «Where is the method which will enable us to say what kind of punishment is justified by absolute morality, and what kind is not?» (pp. 162-163); «Where is the basis of our right to coerce the criminal? (p. 165)»; «What is the legitimate extent of the coercion?» 
Pour Spencer, il y a deux principes fondamentaux dictés par la moralité absolue qui autorisent la coercition. Le premier exige le dédommagement ou la compensation, c'est-à-dire qu'il demande que l'on répare, dans la mesure du possible, le mal que nous avons fait:

(...) the first thing to be required of the transgressor is, that he shall put matters as nearly as may be in the state they previously were. The property stolen shall be restored, or an equivalent for it given. Any one injured by an assault shall have his surgeon's bill paid, compensation for lost time, and also for the suffering he has borne (Spencer, 1860, p. 166$)^{7}$.

Le deuxième principe autorisant la coercition est celui de la protection (générale) dans le cadre d'une intervention minimale. Ce principe vise à prévenir, lorsque cela se justifie raisonnablement, le risque de nouvelles agressions (graves). Mais Spencer souligne à maintes reprises que la moralité absolue n'autorise aucune autre forme de restriction:

(...) no gratuitious inflictions of pain, no revengeful penalties(...) We must permit him to live as completely as consists with social safety. It is commonly said that the criminal loses all his rights. This may be so according to law, but it is not so according to justice (pp. 166-167).

Spencer signale que l'obligation du dédommagement n'a rien de bénin ou de léger: "while equity forbids us to punish the criminal otherwise than making him suffer the natural consequences (exigées par le dédommagement), these, when rigorously enforced, are quite severe enough». Et ceci est d'autant plus vrai qu'il pense qu'en raison de la pauvreté du justiciable le dédommagement va obliger le recours à l'emprisonnement dans la grande majorité des cas.

Suivant la conception de Spencer, la prison ne devrait avoir que deux finalités: une finalité première de mesure auxiliaire du dédommagement (qui tendrait probablement à diminuer avec l'amélioration des conditions de vie des classes les plus pauvres) et une finalité (marginale) de protection dans un nombre limité de cas (et pendant une période également limitée). Mais, selon lui, à son époque, la prison serait presque inévitable parce que très peu de gens seraient en mesure de dédommager les victimes. C'est probablement pour cette raison qu'il ne présente pas explicitement le dédommagement comme une véritable alternative à la prison.

Par ailleurs, compte tenu de ces finalités éthiques et sociales de caractère général, et de ses distances vis-à-vis les théories de la peine, la prison ne

7. Ce principe fut soutenu de manière plus précise et actuelle par Del Vecchio (1951). 
devrait imposer à la personne incarcérée aucune restriction abusive et devrait lui donner, tout particulièrement, l'occasion de travailler, d'être rémunéré (selon les critères de la vie en liberté) et de disposer des fruits de son travail. Spencer juge alors que les limites du droit de punir, en ce qui concerne la qualité des restrictions, sont très claires: rien au-delà du dédommagement ou de ce qui est nécessaire pour l'obtenir ou pour garantir une certaine protection. Il voit cependant une difficulté supplémentaire relative à la détermination de la durée de la peine (ou du quantum) dans les cas de protection. En effet, à ses yeux : «there is considerable difficulty on the duration of the restraint" (p. 180).

Je ne peux pas discuter ici des solutions envisagées par Spencer pour résoudre ce problème. D'ailleurs, en tant que telles, elles présentent peu d'intérêt aujourd'hui et sont souvent insuffisamment explicites, voire parfois contradictoires à certains égards avec ses principes. L'important, c'est l'intérêt qu'il porte à l'ensemble des questions concernant les limites du droit de punir et la priorité qu'il accorde aux principes du dédommagement et de l'intervention minimale. Il est ainsi à la fois en faveur de la modération et contre les décisions artitraires en matière de sanctions, mais il ne cherche pas à obtenir une simple égalité formelle. En effet, pour lui, l'équité doit être recherchée dans l'application de ces deux principes. Je fais peut-être dire ici à Spencer un peu plus qu'il ne dit lui-même, mais je ne crois pas avoir trahi sa pensée.

Il est important d'apprécier comment ce paradigme perdu réussit à poser le problème de l'intervention juridique dans le pénal en accordant la priorité aux mesures de dédommagement, en refusant toute disqualification juridique des personnes trouvées coupables et en prenant en ligne de compte la réciprocité des droits: ceux des personnes condamnées, ceux des victimes directes et réelles, voire ceux, plus abstraits, de la société.

Il faut ensuite insister sur l'autonomie de ce paradigme vis-à-vis l'objectif idéologique de la "guerre à la criminalité», aussi bien que vis-à-vis l'objectif rétributiviste (pénal) de restauration d'une égalité universelle selon la nature des choses (Kant ${ }^{8}$, Hegel; voir Pires, 1990a). En choisissant de ne pas faire appel aux théories de la peine et aux objectifs répressifs du droit criminel, Spencer a réussi à proposer un modèle qui met sur un pied d'égalité les parties impliquées dans les conflits (sans les disqualifier) et qui accorde une place réellement centrale et intégrée aux principes de la priorité du dédommagement et de l'intervention minimale dans la vie des gens.

Bien sûr, Spencer valorise beaucoup les efforts faits en prison pour rendre le système plus souple, pour améliorer le sort des personnes incarcérées et

8. Voir les remarques de Villey $(1979$, p. 23) sur Kant. 
pour les assister le plus possible. Mais ni la réadaptation, ni la dissuasion, ni la neutralisation (au sens précis de cette théorie) ne figurent dans les principes justifiant la sanction. Bien au contraire, la réadaptation fait partie seulement des expériences pratiques qui ont été mises en œuvre sans référence aux principes éthiques. C'est d'ailleurs pour cette raison que l'on oublie, entre autres choses, les droits des justiciables. Avec quelques modifications, ce que Spencer dit ci-dessous à propos de la réadaptation s'applique aussi aux autres théries de la peine:

On the one hand, neither in the doctrines of pure equity with which we set out, nor in the corollaries drawn from them, is there any mention of criminal reformation: our concern has been solely with the rights of citizens and convicts in their mutual relations. On the other hand, those who have carried out the improved penal systems above described, have had almost solely in view the improvement of the offender: the just claims of society, and those who sin against it, having been left out of the question (Spencer, 1860, p. 178).

\subsection{LE PARADIGME «DE L'ÉTHIQUE TOTALE» (OU DE LA GUERRE CONTRE LA CRIMINALITÉ)}

Pour faciliter la comparaison, j'illustrerai ce paradigme à l'aide des idées de Garofalo $(1887$; 1914) à l'égard du dédommagement et, tout particulièrement, de sa communication au Congrès pénitentiaire de Bruxelles en 1900 dont le titre a une saveur d'actualité: Enforced Reparation as a Substitute for Imprisonment.

Premièrement, il est intéressant de noter comment ce paradigme même, lorsqu'il parle de dédommagement, accorde une place centrale à l'objectif de «guerre à la criminalité». Le point de départ de Garofalo est donc bien différent de celui de Spencer. En effet, considérons la manière dont il a justifié le recours au dédommagement. Il invoque d'abord des raisons qui relèvent d'une «économie punitive»: 1) cette mesure permettrait de résoudre le problème de la surpopulation carcérale qui empêche les prisons d'individualiser le traitement et donc d'aboutir à de meilleurs résultats; 2) l'effet de dissuasion du dédommagement serait, à ses yeux, plus fort que celui des courtes peines d'emprisonnement; 3 ) cette mesure aurait l'avantage supplémentaire d'être moins onéreuse pour le contribuable. À ces considérations, Garofalo ajoute une raison d'ordre éthique: la protection des droits légitimes des victimes. Mais même ces droits sont présentés d'une manière problématique. Car tout se passe comme si les droits des justiciables n'existaient pas ou n'étaient pas importants. Contrairement à Spencer, Garofalo justifie alors en bonne partie 
cette sanction par référence aux théories de la peine et il conçoit le dédommagement exclusivement comme un mécanisme bienfaisant de répression et de contrôle.

Remarquons que Garofalo fait appel à la théorie de la dissuasion pour justifier le dédommagement, qu'il considère comme une "new form of repression" $(1914$, p. 227). C'est qu'il partage cette triple stratégie de la criminologie positiviste à l'égard de la «guerre à la criminalité»: réadapter quand cela est possible, éliminer ou neutraliser ce que l'on ne peut pas réadapter et prévenir ce qu'il est possible de prévenir (Garland, 1986, pp. 95-96). Or, la théorie de la dissuasion n'est pas incompatible avec ces objectifs:

And the foreclosure of such a possibility will be a much more potent means of disarming the enemies of society than the ridiculous punishments which are today imposed in the hope of reforming offenders (Garofalo, 1914, p. 343).

(...) means of prevention much more potent than the menace of briefterms of imprisonment (Garofalo, 1900, p. 423).

Deuxièmement, il faut relever les omissions, ce dont Garofalo ne parle pas. Il ne parle pas, par exemple, des droits des personnes condamnées. Et moins encore d'équilibre ou de réciprocité des droits. Bref, l'objectif de Garofalo n'est même pas «tempéré» par la considération des droits des justiciables. Hors les cas extrêmes, rien ne semble inquiéter le criminologue ou le pousser à vouloir limiter le droit de punir de l'État. Garofalo va proclamer à voix haute la nécessité de défendre les droits des victimes, ce qui est sans doute "progressiste»: «the insufficiency of the protection accorded to the injured person is everywhere recognized in an equal degree» (Garofalo, 1900, p. 421). Mais il prendra bien soin de ne pas mentionner les droits des personnes condamnées. Voici donc un des sens que je donne à l'expression d'éthique totale: elle s'enferme autour du justiciable sans le considérer vraiment comme un sujet des droits; il est une "cible" de l'intervention et celle-ci vise à extirper la «criminalité» qui existe en lui.

Lorsque nous affirmons aujourd'hui que la criminologie a négligé les droits des victimes, nous avons sans doute raison; en revanche, lorsque nous affirmons, ou laissons entendre, que la criminologie s'est préoccupée davantage des droits des personnes condamnées, c'est carrément inexact: la criminologie s'est occupée des personnes incarcérées, mais non de leurs droits. La différence n'est pas négligeable.

Résultat: Garofalo ne se soucie aucunement des limites du droit de punir, de l'équité, des questions relatives à la détermination de la longueur des peines d'emprisonnement, etc., bref, de toutes les questions concernant la qualité 
et la durée des restrictions imposées aux personnes. L'objectif principal ici est l'élimination ou la réduction radicale de la criminalité à travers notamment une politique pénale. Et celle-ci est conçue comme une politique de guerre.

Enfin, il est étonnant de voir comment Garofalo réussit à présenter une mesure, en soi progressiste, d'une manière et avec un langage extrêmement répressif. De quoi désenchanter n'importe quel partisan de la modération. Certes, pour Garofalo cette mesure n'est jamais exclusivement un dédommagement comme elle peut l'être pour Spencer. Elle a deux volets : une indemnisation payée à la victime et une amende payée à l'État. Mais ce n'est pas cela qui lui donne une allure répressive. C'est son langage, l'absence de considérations d'ordre éthique et juridique à l'égard de toutes les parties concernées et les mécanismes juridiques qu'il préconise pour assurer ce dédommagement".

Par contre, il est déroutant de voir comment, malgré ses positions partisanes, Garofalo peut aller loin dans la portée qu'il souhaite donner au dédommagement si l'on considère exclusivement les types d'infractions. Cette mesure peut s'appliquer au cas de mort et de blessures par négligence, aux voies de fait, à la diffamation, au vol non qualifié, à certains types de fraude, aux dommages volontaires à la propriété, etc. (Garofalo, 1914, pp. 227, 389-391). Il revendique aussi la création d'un fonds d'indemnisation pour les victimes qui serait alimenté en partie par l'argent obtenu par les amendes. Et il affirme même: «I would confine the use of the prison within comparatively narrow limits» (p. 425). Mais, encore là, cette recommandation n'est dictée que par un souci d'efficacité.

Il n'est donc pas étonnant de constater que les mouvements pour les personnes incarcérées aient insisté sur la question des «droits» à part entière de ces personnes. C'est que le programme criminologique, trop axé sur le but pragmatique de «combattre la criminalité», cette entité métaphysique que le criminologue croyait «enracinée» dans l'individu (Garland, 1986), proposait un modele d'intervention sans droits et sans principes éthiques suffisamment explicités.

9. Garofalo propose un système complexe - voire confus - de mesures de différentes sortes pour assurer la réparation : avant le jugement, pendant le jugement et après le jugement. Ces mesures visent, entre autres choses, à prévenir toute tentative de la part de l'accusé d'escamoter ses biens pour ne pas payer ses dettes, et à résoudre le cas des justiciables qui ne disposent pas de biens ou d'argent, etc. 


\section{L'ÉVOLUTION DE LA PROBLÉMATIQUE DES DROITS DES PERSONNES INCARCÉRÉES AU QUÉBEC}

L'histoire détaillée la défense des droits des personnes incarcérées au Québec reste à faire ${ }^{10}$. Si, sur la base de nos connaissances lacunaires, je devais faire une hypothèse sur le «dossier principal» de la lutte pour les droits des personnes incarcérées au Québec pour toute la période entre 1963 et 1980, je dirais ceci : il s'agit de la lutte contre la «politique du secret» ou «des portes closes» de la prison. Lutte qui visait à la fois à exercer un droit de regard sur ce qui se passe à l'intérieur des murs et à pouvoir informer le public d'une manière non officielle. Bref, c'était une lutte pour l'accès à l'information et pour l'éducation du public sur la vie en prison.

La lutte contre la construction de l'Unité spéciale de détention (USD) (dès 1965), ou pour la fermeture de Parthenais (non obtenue), ou pour de meilleurs soins médicaux, ou pour l'éducation des analphabètes, ou pour éviter que les jeunes ne soient incarcérés avec les adultes n'ont été que des dossiers ponctuels, bien que tout cela, et beaucoup plus, ait pris aussi le temps et l'énergie de ces personnes.

À titre d'hypothèse, $\mathrm{j}$ 'aimerais retenir quatre moments et lignes de force de l'évolution de la question des droits des personnes incarcérées au Québec, à partir de la première moitié des années 1960:

i. Tout d'abord, le mouvement qui naît au Québec autour de la Ligue des droits de l'homme va prendre en ligne de compte, entre 1965 et aujourd'hui, des questions de nature fort diversifiée: soins psychiatriques et médicaux appropriés, séparation des jeunes incarcérés d'avec les adultes, abolition des «traitements» inhumains dans des unités spéciales, etc. Mais aussi : lutte pour l'abolition de la peine de fouet et de la peine de mort dans le Code criminel, lutte pour une meilleure Loi du Coroner, pour le droit de regard dans les prisons, pour un moratoire sur la construction des prisons, etc. Bref, des questions relevant à la fois des droits-résistances, des droits-créances (services) et des questions de réforme pénale. Le rapport de l'enquête sur les conditions de détention entreprise par l'ODD, Les Prisons de par ici (Landreville, Gagnon, Desrosiers, 1976) - un ouvrage dont l'importance sociale pour la justice pénale québécoise n'est pas encore suffisamment reconnue - reflète bien cet intérêt diversifié pour les droits-résistances et les droits-créances. Mais aussi l'importance capitale de cet objectif premier qui consiste à «percer ce mur du silence et de tenir le public au courant» (p. 11).

10. Nous disposons par ailleurs d'une monographie sur le rôle de la Ligue des droits de l'homme du Québec (LDH) (Laurin, 1985). Dans cette étude, un chapitre est consacré à l'Office des droits des détenus-e-s (ODD). 
Les juristes jouent un rôle important non seulement sur le plan de revendications, mais aussi en offrant leurs services comme médiateurs dans les conflits et en essayant de bâtir un droit carcéral capable de faire sortir les pratiques correctionnelles de l'ombre et de limiter le pouvoir correctionnel de gestion qui avait pris l'habitude de fonctionner sans avoir vraiment à rendre compte à personne de ses décisions. L'Aide juridique participera en mettant sur pied, pendant une certaine période, un service de droit carcéral " ${ }^{1}$. Cette recherche d'un droit carcéral, voire correctionnel, se poursuit encore de nos jours (Lemonde, 1990).

ii. Ensuite, la pensée criminologique se modifie et devient de plus en plus critique vis-à-vis son propre passé et le système pénal; les questions de réforme du droit commencent à prendre de l'ampleur à la suite des premières publications de la Commission de réforme du droit, dans les années 1974-1976; et les questions constitutionnelles au Canada en matière de droits de la personne prennent un nouvel élan (particulièrement après 1980). On assiste alors à un élargissement et à une bifurcation d'intérêts qui étaient auparavant imbriqués. D'une part, les questions relatives au droit carcéral, traitées jusque-là d'un point de vue essentiellement juridique, relèveront de plus en plus des droits de la personne (voir Lemonde, 1990). D'autre part, on observera une tendance chez les criminologues à tracer les grandes lignes de la réforme carcérale sur la base d'une critique d'ensemble du droit pénal, sans toutefois abandonner complètement la dimension des services aux personnes incarcérées et leurs conditions de vie. Ces deux aspects de la question demeurent cependant complémentaires à certains égards.

Nous pouvons peut-être discerner deux grandes périodes de cette évolution. Celle qui va du début des années 1960 à 1975 environ se caractérise par l'accès à la prison et par l'énonciation des droits des personnes incarcérées. La période qui commence en 1975 élargit et modifie les termes de la problématique. $L$ 'accent est mis sur la réforme pénale globale, sur les coûts sociaux du système pénal, sur une remise en cause de la réadaptation, sur les mesures de rechange à la prison et l'examen des options abolitionnistes et sur une extension accrue des droits de la personne aux justiciables. Pendant cette période, on relance, avec plus de force, une série de questions qui avaient déjà été abordées: le casier judiciaire, l'emprisonnement par défaut de paiement d'une amende, la violence policière, la longueur excessive des peines, la peine de mort, les conditions de vie en prison et ses effets, le pardon, etc.

Le passage de la publication Les Prisons de par ici, qui caractérise la première période, au programme de recherches autour de la problématique

11. À ma connaissance, Me Robert Sacquitelle a joué un rôle important dans ce projet. 
des coûts sociaux du système pénal ${ }^{12}$ illustre bien ce déplacement d'une partie de la pensée criminologique franco-canadienne. Il s'est manifesté aussi dans les journaux des personnes incarcérées (Le Tremplin, Face à la justice) et dans une série d'organisations «de base» (comme le Comité pour le développement des alternatives à l'incarcération de la ville de Québec) ${ }^{13}$ à partir de 1975.

iii. La troisième tendance est liée au débat sur les théories de la peine. Elle est très complexe et ne peut faire l'objet d'un développement ici. Qu'il suffise de signaler que la question des droits des personnes incarcérées a entretenu, au début, des rapports ambivalents avec la théorie de la réadaptation. Lorsqu'il s'agit de demander des «services» (droits à...), certains praticiens favorables au mouvement pour les droits font quand même référence au principe de réadaptation, tandis que les chercheurs et d'autres praticiens ont tendance à revendiquer ces mêmes services exclusivement au nom des droits (Landreville et al., 1976, pp. 16, 117-118). Par ailleurs, la période entre 1975 et 1985 se caractérise par un désenchantement marqué à l'égard de la théorie de la réadaptation, mais aussi par des hésitations et des doutes sur ce qui serait susceptible de la remplacer. Les prises de position sont alors souvent tâtonnantes et variées. Bref, on essaie de trouver des nouvelles assises pour penser les sanctions et justifier les mesures de rechange à la prison. Après 1985, on voit renaître l'intérêt pour la relation d'aide et pour la dimension des «services» qui avait été quelque peu éclipsé dans la période entre 1975 et 1985 en raison de l'accent mis sur la critique du principe de réadaptation.

iv. La demière tendance est le développement explicite et spécifique d'un souci à l'égard des droits des femmes incarcérées sous l'impulsion et l'influence du mouvement féministe. Bertrand (1979) a joué un rôle pionnier dans l'articulation des perspectives féministes avec la criminologie francocanadienne. Il y a eu également, par la suite, une fusion des perspectives féministes avec la problématique des coûts sociaux du système pénal pour les femmes (Hamelin, 1989; Poisson, 1988). Du point de vue des revendications pratiques, certains progrès ayant déjà été obtenus au chapitre des droitsrésistances, l'accent fut ici mis d'emblée sur les droits-créances. Car l'état des services et des conditions de détention pour les femmes était déplorable en comparaison du sort réservé aux hommes. Cette lutte pour de meilleurs

12. Ce programme débute en 1975-1976 à l'École de criminologie autour de Pierre Landreville (Landreville, Blankevort et Pires, 1980) et il se poursuit tout au long des années 1980 sous la direction de Landreville, à l'Université de Montréal (voir Hattem, 1980, 1984, 1987, 1991; Pires, 1983; Hamelin, 1989), et sous ma direction, à l'Université d'Ottawa (voir Poisson, 1988; Rochon, 1988; Arsenault, 1989). Voir aussi Hattem et Parent (1982).

13. Ce comité a produit la brochure «Dire non c'est logique!» pour exprimer son désaccord face à la construction de nouveaux pénitenciers au Québec. 
services aux femmes a contribué à son tour, me semble-t-il, à cette revalorisation générale de l'idée de services et de relation d'aide après 1985.

\section{LE DÉSENCHANTEMENT À L'ÉGARD DU PROJET PÉNITENTIAIRE}

Pour bien comprendre ce qui a motivé les orientations abolitionnistes d'une partie des mouvements pour les droits des personnes incarcérées aussi bien que les déchirements existentiels que ces développements ont provoqués, il faut évoquer brièvement le contexte de crise socio-culturelle de la fin des années 1960 et des années 1970 (Pires, 1983, pp. 7-14). Deux axes sont particulièrement importants: les événements (par exemple les émeutes) et critiques concernant la prison et la prise de conscience d'autres formes d'oppression (à l'égard des femmes, des minorités ethniques, des populations enfermées, etc.) que celle qui frappe la classe ouvrière. Cette prise de conscience est à la base des mouvements qui se sont développés en Amérique du Nord pour les droits des groupes de «moindre pouvoir».

Le désenchantement à l'égard du projet pénitentiaire fut donc caractérisé par la convergence d'événements et de facteurs de différents ordres: ainsi, la surreprésentation des pauvres et des minorités en prison apparait désormais comme un effet discriminatoire du système pénal, la réadaptation et le programme positiviste font l'objet d'une remise en question et le champ symbolique des droits de la personne englobe désormais les justiciables, etc.

Dans ce contexte, certaines personnes intéressées par la réforme prennent conscience, notamment à travers quelques recherches historiques ${ }^{14}$, d'un fait à la fois intrigant et, pour certains, révoltant. On a pu constater que le grand constat d'échec de la prison fait dans les années 1970 n'était que la pointe d'un énorme iceberg et qu'il n'était pas du tout aussi «révolutionnaire» qu'on l'avait cru. En effet, ces recherches nous ont fait remarquer qu'en lisant les documents officiels produits en différents pays depuis le XIX ${ }^{e}$ siècle, on constate ceci : la critique de la prison était contemporaine de sa naissance. Entre 1820 et 1845, on dénonce déjà le pénitencier comme le grand échec de la justice pénale. Et cette critique devient de plus en plus incisive au fur et à mesure que l'on s'approche du tournant du siècle. En outre, les chercheurs ont souligné que cette critique était répétitive et monotone (Foucault, 1975,

14. La bibliographie est considérable. Voir particulièrement les travaux de David Rothman $(1971,1975,1980,1981)$ et de Michel Foucault $(1975,1976)$. Voir aussi Rusche et Kirchheimer (1939, p. 105). 
pp. 269-273 ${ }^{15}$ ). On dira, par exemple, que la prison est une école du crime et qu'elle produit (en partie) la récidive, etc.

Mais ce n'est pas tout. Les recherches ont souligné que ce grand constat d'échec, pour étonnant que cela puisse paraître, n'a pas débouché sur une mise en cause globale de l'emprisonnement. On va alors critiquer tel ou tel aspect de la prison sans la rejeter comme telle (Foucault, 1975, pp. 269-275; Rothman, 1971, p. 93; 1975, p. 18). Quoi qu'il en soit, tous les réformateurs - même ceux qui sont favorables à une discipline draconienne - dénoncent plus ou moins sévèrement certains aspects de la vie matérielle en prison (par exemple la qualité parfois «clairement» déplorable de l'alimentation) et l'absence d'un bon système de classification. Et tous sont d'accord sur l'échec de la prison. Ceux qui veulent que la prison reste dissuasive croient qu'elle pourrait mieux réussir si elle prenait soin de ne pas imiter le mode de vie de la société, si on parvenait à faire respecter une discipline stricte, si on limitait les contacts des personnes incarcérées avec le monde extérieur, si on ne pouvait en aucun cas abréger la durée des peines, etc. Par contre, ceux qui veulent que la prison devienne plus correctrice croyaient qu'elle pourrait réussir $s i$ les conditions en institution étaient plus saines, si un certain nombre de programmes étaient mis sur place, si le travail en prison était stimulant et rémunérateur, si on avait un bon directeur, si on réussissait à combler le fossé qui sépare les gardiens des personnes incarcérées, si on réussissait à modeler la vie carcérale davantage selon les critères de la vie en société, etc. Les réformateurs craignent encore plus un monde sans prisons qu'un monde avec des prisons inutiles et mauvaises (Rothman, 1981, pp. 378-379).

Pour Rothman, cette foi en la prison est particulièrement frappante, compte tenu de toute la misère humaine et la violence dont les réformateurs du $\mathrm{XIX}^{e}$ siècle font état dans leur description de la prison. En effet, quiconque a lu un de ces rapports d'enquête du $\mathrm{XIX}^{\mathrm{e}}$ siècle sur les conditions de vie en prison peut se retrouver sans peine dans les remarques de Rothman (1981, p. 378):

On lit les enquêtes de 1880 et 1890 sur la brutalité en prison avec une certaine tension. Comment ce rapport législatif va-t-il conclure? Sera-t-il capable de passer par-dessus toute cette misère et de proposer encore une recommandation visant à perpétuer le système? Invariablement la réponse sera «oui». Même beaucoup de temps après qu'il devient évident que la prison n'est pas en mesure d'accomplir les nobles objectifs de ses

15. Les recherches canadiennes faites par la suite ont abouti sensiblement aux mêmes résultats (Pires, 1986; C.C.D.P., 1986; Laplante, 1989). 
fondateurs, l'emprisonnement continuera à exister. (C'est nous qui traduisons.)

Un bon nombre de ceux qui critiquaient les prisons dans les années 1970 ont été bouleversés par la prise de conscience de cette capacité du système carcéral à absorber les critiques sans se transformer fondamentalement. Comment expliquer la résistance de la prison à toutes ces critiques? Et comment ne pas répéter les erreurs des réformateurs précédents? Certes, plusieurs hypothèses ont été évoquées pour expliquer cette résistance de la prison aux changements de fond. Je ne peux pas les présenter ici. À dire vrai, aucune de ces hypothèses ne me semble entièrement satisfaisante et certaines encore moins que d'autres. Je veux tout simplement souligner le poids de cet énorme consensus qui s'est formé autour de l'échec de la prison et de la nécessité de faire quelque chose de différent.

Il faut remarquer que ce consensus fut le fait de gens avec des visions du monde et des insertions professionnelles bien différentes ${ }^{16}$. C'est dans cette conjoncture générale que les mouvements de réforme ont eu à se prononcer sur une nouvelle stratégie à adopter. Ils voulaient à tout prix éviter de commettre les mêmes «erreurs» que les commissions et les rapports précédents en demandant, encore une fois, une meilleure prison ou de nouvelles prisons. C'est alors qu'on a beaucoup discuté, au Québec, des hypothèses de Foucault et des orientations de réforme proposées par Louk Hulsman $(1979,1982)$ et par Thomas Mathiesen (1974). Ce dernier était fortement engagé dans un mouvement pour les droits des personnes incarcérées dans les pays scandinaves et avait classifié les réformes en deux types: les réformes positives, qui visent à améliorer et à développer une institution, et les réformes négatives, qui visent à réduire, voire à abolir une institution.

En ce qui concerne les prisons et le système pénal dans son ensemble, Mathiesen recommandait à tous de s'engager exclusivement dans les réformes négatives. Car l'expérience avait démontré que les propositions positives ne faisaient que légitimer et reproduire le système sans le modifier fondamentalement. Les réformateurs modérés et de "gauche» étaient tous d'accord pour dire qu'il fallait mettre un terme aux «politiques expansionnistes» et faire quelque chose de différent.

Se pose alors le dilemme suivant: Qu'est-ce que veut dire au juste «ne pas faire de réformes positives»? Doit-on arrêter de demander de meilleures conditions de vie en prison pour éviter de reproduire le système? Certes, ce dilemme se pose aussi devant certaines formes de charité. Ainsi, lorsqu'on

16. Voir, par exemple, le point de vue de Mattick (1973) qui compte vingt ans d'expérience dans le domaine de la justice criminelle. 
donne de la monnaie à un mendiant, on se demande parfois si on «reproduit un système» ou si on «aide une personne». Et nous sommes rarement cohérents dans nos choix: un jour nous décidons de ne pas donner "pour ne pas reproduire le système» et le lendemain de donner "pour aider une personne" (même si de ce fait l'on reproduit le système). De même, au Québec, les positions sont demeurées incohérentes: on affirmait un principe un jour et on pratiquait le contraire le lendemain. D'ailleurs, je ne connais aucun mouvement pour les droits des personnes incarcérées au Canada qui ait adopté de manière stricte le principe de Mathiesen. Bien au contraire, on a fait des recommandations qui allaient à l'encontre de ce principe et qui visaient, entre autres choses, à l'amélioration des conditions de vie en prison (voir Landreville, Blankevoort et Pires, 1980). En outre, les positions de Mathiesen ont été moins influentes au Québec que celles de Hulsman et de Foucault, qui étaient beaucoup moins radicales à cet égard.

Cependant, il y a eu pendant cette période (1970-1985), particulièrement aux États-Unis, un glissement de sens important qui nous a affectés en partie, soit une certaine tendance à confondre les problèmes que pose la prison avec ceux que pose la théorie de la réadaptation. Or, s'ils sont reliés, ils ne sont pas identiques. Bref, on a commencé à attribuer à celle-ci tous les problèmes de celle-là. Certains sont venus à penser alors, dans le feu de l'action, qu'on pouvait trouver dans l'idée de punition proportionnelle et modérée (von Hirsch, 1976) une solution au problème de l'usage abusif de la prison. C'est ainsi qu'aux États-Unis, on a proposé la théorie du juste dû ou encore le retour au rétributivisme classique comme mesure de rechange au modèle de la réadaptation. Ces autres modèles ne nous offrent cependant pas une solution convenable (Pires, 1990a). Mais que faire alors? Insister sur l'idée de réadaptation? Condamner l'idée même de mesures de rechange à l'incarcération et prôner un retour à la prison?

Les positions prises par une partie de la criminologie franco-canadienne ont été les suivantes. Afin de ne pas se laisser traîner sans résistance là où on ne veut pas aller tout en restant à l'écoute des besoins humains immédiats, il a fallu accepter d'abord le risque de l'incohérence et de la récupération et, ensuite, garder une orientation de réforme favorisant le principe de l'intervention minimale en matière de droit criminel. Par rapport à la prison, on a alors distingué grosso modo deux types de problèmes: celui relatif au nombre de places dans les prisons (et la durée des peines) et celui du régime en vigueur dans les prisons. Par rapport au premier, la règle générale est encore celle de Mathiesen. Ainsi, il faut demander un moratoire dans la construction de nouvelles prisons afin d'empêcher que cette voie ne soit adoptée pour résoudre le problème séculaire de la surpopulation. De même, l'ouverture de nouvelles prisons plus adaptées et modernes doit être effectivement accompagnée de 
la fermeture des vieilles, etc. S'ajoutent à cela les efforts pour réduire la longueur des peines.

Par rapport au régime de vie en prison, je crois qu'il ne faut pas hésiter: il faut l'améliorer, même si cela n'a rien en soi de «progressiste». C'est d'ailleurs depuis longtemps l'avis de Hulsman et aussi de Foucault (1976, p. 27):

Faire régresser la prison, diminuer le nombre des prisons, modifier le fonctionnement des prisons, dénoncer tous les illégalismes qui peuvent s'y produire... Ce n'est pas mal, c'est même bien, c'est même nécessaire.

Ce qui me semble aujourd'hui important, pour paradoxal que cela puisse paraître, c'est de ne pas se donner comme principal objectif de reforme la réduction de la «criminalité», mais de viser plutôt une hausse de la qualité de vie pour l'ensemble de la population et l'amélioration de notre système de résolution de conflits, ce qui pourrait permettre de diminuer le nombre de certaines situations-problèmes ou, tout au moins, de compter sur un système de résolution de conflits moins côteux socialement. Car cet objectif de «guerre à la criminalité» évoque trop rapidement à l'esprit une représentation mystifiée des personnes et des problèmes, les formidables «théories de la peine» et des méthodes passablement répressives. Je pense, en outre, qu'il faut étudier l'échec des mesures de rechange afin de mieux orienter les propositions visant à une réduction radicale de l'incarcération. Il faut aussi éviter l'adoption de la théorie du juste dû, entre autres raisons parce qu'elle ne fait pas (encore) une place adéquate à la déjudiciarisation et aux mesures de rechange à l'incarcération (Pires, 1990a).

Spencer concluait son article de 1860 d'une manière qui n'est peut-être pas optimiste, mais qui peut nous aider à réfléchir sur ce qui a commencé à se produire chez nous après le bouillonnement d'idées nouvelles de réforme de nature humaniste qui ont marqué les années 1970 et le début des années 1980. Il écrivait alors ceci:

J'ai donné occasionnellement libre cours, avec certaines réserves, au paradoxe selon lequel l'humanité ne prend le droit chemin qu'après avoir essayé tous les moyens possibles pour aller dans la mauvaise direction. J'ai observé cependant récemment qu'à certains égards ce paradoxe ne disait pas encore toute la vérité. Car certaines occasions m'ont fait voir que même lorsque l'humanité avait enfin trouvé la bonne direction, elle retournait souvent délibérément à la mauvaise. (Spencer, 1860, pp. 190-191) (C'est nous qui traduisons.) 


\section{GRANDEURS ET MISÈRES DES HUMANISMES DANS LES THÉORIES DE LA PEINE}

Lorsqu'on examine les discours justifiant les peines, on constate un débat curieux. Les théoriciens de la peine se livrent en effet à un concours paradoxal: d'une part, c'est à qui se montrera le plus humaniste ou le plus respectueux de certaines valeurs; d'autre part, ils s'accusent réciproquement d'humanisme et ils n'aiment pas en être accusés. L' «humanisme» a donc, dans ce débat, un double statut: il est tantôt quelque chose d'enviable, une valeur noble à conserver, la preuve que nos sociétés ne sont pas barbares, bref un "avantage»; tantôt indice de mollesse, de sentimentalitê, voire d'une certaine lâcheté, donc un «désavantage». On essaie de faire un tri entre le «bon» et le "mauvais» humanisme. On veut savoir laquelle des théories est la plus humaine et, en même temps, laquelle des théories est la plus ferme, la moins débile ou «efféminée" (Beaumont et Tocqueville, 1845, p. 136), celle qui nous détourne le moins dans notre route ou de nos principes absolus. C'est que l'humanisme est, dans les théories de la peine, à la fois chanté et maudit. Le dilemme de chaque théorie de la peine est donc le suivant: comment être humaniste sans "céder» à l'humanisme?

Avant de faire un rapide survol de ce curieux débat, il convient de se rappeler que le souffle humaniste, aussi authentique qu'il soit, ne permet pas, hélas, d'éviter des erreurs. Cela veut dire deux choses: si quelqu'un se trompe, on ne peut pas en inférer qu'il n'avait pas d' «intention humaniste»; à l'inverse, si une personne a des «intentions humanistes», cela ne veut pas dire qu'elle ne se trompe pas. En effet, les bonnes intentions et les humanismes ne constituent pas un garde-fou contre les erreurs et ne préviennent pas nécessairement des développements fâcheux.

Ensuite, il faut se rappeler que la situation est encore plus périlleuse lorsqu'on accepte d'«enfermer» nos humanismes dans des limites qui sont au départ problématiques. Or, les théories de la peine ont une fâcheuse tendance à coloniser notre imagination et à enfermer notre humanisme dans des limites passablement étroites. On est alors souvent appelé à trancher une alternative de ce genre-ci: qu'est-ce qui est le plus humain ou le plus conforme à nos mœurs: administrer le fouet ou imposer une longue réclusion? Ces théories nous amènent à s'imaginer dès le départ qu'il est juste ou utile de faire mal et d'imposer une "peine» (au sens du droit criminel); elles nous demandent ensuite de décider, avec une marge de manœuvre assez réduite, ce qu'est un mal qui n'est pas abusif; bref, de faire la différence, souvent trop subtile, entre «se venger» et «honorer les gens en leur donnant la peine méritée», entre «punir» et «envoyer les gens en prison pour les traiter», etc. 
Examinons la position du rétributivisme pénal dans ce concours paradoxal sur les humanismes. Il se veut bien sûr humaniste. Car il pose l'exigence de la proportionnalité de la peine (sur la forme de la loi du talion): le châtiment ne peut donc, sous aucun prétexte, excéder la faute. De plus, il soutient que l'être humain ne doit pas être un instrument pour des fins d'autrui, ni au nom de «son propre bien». En outre, le rétributivisme se prétend humain parce que la peine «juste» est une manière d' «honorer» le coupable.

Parallèlement, cette théorie se dit aussi ferme et peut bien se défendre contre toute «accusation» d'humanisme. Ainsi, un philosophe (bumaniste) de l'envergure de Kant (1796, p. 216) a justifié l'application de la peine de mort indépendamment de tout critère d'utilité : même si la peine de mort est clairement inutile, le dernier meurtrier en prison doit être exécuté au nom du respect de nos principes moraux. Plus important encore: pour cette théorie, la punition est un devoir moral impératif en matière pénale : toute autre forme de résolution du conflit est ici condamnée et le pardon n'est que rarement recevable. On voit que cette théorie remplit toutes les conditions d'une «bonne» théorie de la peine : elle est humaine et, cependant, personne ne peut l'«accuser» de l'être.

La théorie de la dissuasion remplit aussi ces conditions. En effet, elle est humaniste puisqu'elle soutient aussi, à sa façon, que les peines doivent être proportionnelles aux délits (Beccaria, 1764, p. 48): il ne faut appliquer ni les moindres châtiments aux plus grands crimes ni les grands châtiments aux délits triviaux. Elle s'élève ensuite contre tout excès despotique du pouvoir de punir: excès d'arbitraire et excès des peines. Elle critique les «abus d'un pouvoir sans bomes» et cette "froide atrocité que les hommes puissants regardent comme un de leurs droits» (Beccaria, 1764, pp. 46-47). Pour qu'une peine soit dissuasive, il suffit que le mal qu'elle cause surpasse le bien que le coupable a retiré de son acte. Or, surpasser n'est pas excéder. On doit choisir la moindre des peines applicables dans les circonstances (Beccaria, 1764, p. 159). En outre, la théorie de la dissuasion est humaniste parce qu'elle affirme aussi que la rapidité de l'intervention compte plus que la sévérité de la peine. Plus encore: puisque le seul objectif social de la peine est de détoumer les personnes du crime, si l'on peut attendre autrement cet objectif, la peine classique ne se justifie plus. Le dédommagement peut donc être suffisant. Dès lors, la peine prend un caractère subsidiaire (Jhering).

La théorie de la dissuasion est-elle ferme? Elle se défend bien également sur ce plan. Beccaria s'oppose à la peine de mort, mais ses arguments ne sont pas exclusivement humanistes: «la rigueur du châtiment fait moins effet sur l'esprit que la durée de la peine» (p. 92). D'autres, comme Bentham et Tocqueville, vont «renforcer» cette fermeté de la théorie. Pour ce dernier, 
la peine doit être sévère et le «but (...) de la philanthropie n'est pas de rendre les prisonniers heureux, mais meilleurs» (cité par Perrot, 1984, p. 21).

La théorie de la réadaptation est humaine, bien sûr, parce que beaucoup de ses représentants se sont battus contre les peines sanguinaires d'antan: les châtiments corporels et la peine de mort. Humaine aussi parce qu'elle a critiqué les conditions matérielles inhumaines de la prison au point d'attirer sur certains philanthropes la critique féroce d'un Tocqueville. Humaine encore parce qu'elle a voulu séparer les enfants des adultes, les femmes des hommes, les fous des "criminels", etc. Humaine, d'ailleurs, par son propre idéal de réadaptation morale, psychologique ou sociale des justiciables de milieu défavorisé. Humaine, enfin, parce que dans ses formulations les plus critiques, elle préfère la relation d'aide en liberté que l'enfermement et parce qu'elle perçoit de plus en plus l'importance d'une réflexion éthico-juridique sur les limites du droit de punir et d'une mise en pratique du principe de l'intervention minimale (Ouimet, 1969).

Pourtant, cette théorie s'est présentée aussi, et se présente encore, sous une figure insensible et sévère. Elle a affirmé qu'il ne fallait pas se leurrer sur la vraie nature des «petits contrevenants» ni les jauger à partir de leurs actes apparemment peu graves; que la durée des peines devrait être indéterminée ou assez longue pour permettre un «traitement efficace»; que le temps passé en prison n'était pas aussi perdu ni aussi dramatique que certains se l'imaginent, car l'être humain est une machine merveilleuse: il peut s'adapter «sans dommage grave et irréversible» à presque toutes sortes d'environnements. Et ceci est encore plus vrai pour cette «espèce particulière» que sont les «méchants».

Chaque fois qu'une théorie se présente sous sa figure «sévère» pour répondre aux «accusations» d'humanisme, elle est alors perçue par les autres comme dérapant vers la répression. Retenons un seul exemple. Le rétributivisme reproche à l'utilitarisme de vouloir faire de l'être humain un instrument pour dissuader les autres, pour protéger la société ou pour atteindre des fins imposées par les autres. Voilà, selon le rétributivisme, la dimension répressive de l'utilitarisme pénal. Mais l'utilitarisme reproche à son tour au rétributivisme (kantien) l'attachement de ce dernier au principe selon lequel la peine est un devoir moral et doit toujours être appliquée dans toute sa rigueur, même lorsqu'elle est socialement inutile. Pour les utilitaristes, ce principe représente un gaspillage social «inhumain». La boutade de Jhering (1877, p. 292), qui est favorable à l'utilitarisme, est à propos ici : il disait que si Beccaria n'avait pas protesté contre les peines excessives dans son ouvrage Des délits et des peines (1746), Adam Smith aurait été poussé à le faire en traitant des Causes de la richesse nationale (1776) afin de prévenir la société de sacrifier, sans 
nécessité, le capital de vie et de travail d'un de ses membres. Bref, le rétributivisme pénal est contre l'usage utilitaire de la peine, mais en faveur (par principe) de la punition inutile; l'utilitarisme pénal, en revanche, est en faveur de l'usage utilitaire de la peine, mais contre la punition inutile.

Et on vous demande de choisir! Comme écrivait à juste titre un éminent juriste et philosophe du droit italien, ce qu'il y a de mieux dans les théories de la peine c'est les critiques qu'elles s'adressent les unes aux autres (Del Vecchio, 1951, p. 211). Il aurait peut-être dú ajouter l'avertissement suivant: prenez garde, en essayant de les combiner, vous risquez de perdre justement ce qu'elles ont de meilleur!

$\mathrm{Si}$, le dos au mur, je devais faire un choix (aujourd'hui, au Canada) en fonction de ces miettes humanistes des théories de la peine et de leur capacité à se modifier pour devenir autre chose, je choisirais l'humanisme de la théorie de la réadaptation. Mais ce choix serait qualifié en dénonçant à haute voix toutes les autres "peccadilles». Dans le cas de la théorie de la réadaptation, il s'agirait de dénoncer en même temps la conception du justiciable comme un être "anormal», l'idée de sentences indéterminées, l'attachement outrancier que certains représentants de cette théorie ont encore pour la prison, leur manque d'intérêt pour les questions d'éthique et de limites de l'intervention dans la vie des gens, etc. Bref, il resterait peut-être très peu de ce qui fut un jour la théorie elle-même.

À l'instar de ce qui s'est produit dans d'autres secteurs de la vie sociale et universitaire, les mouvements pour les droits des personnes incarcérées ont éclaté dans toutes les directions et il est très difficile de porter un jugement d'ensemble sur les orientations de ces groupes et des chercheurs qui y contribuent sur le plan théorique. Certains auteurs accordent beaucoup de valeur aux positions abolitionnistes, d'autres non; certains auteurs ont beaucoup d'espoir dans la capacité du droit formel et des tribunaux de résoudre les problèmes de la prison, d'autres non; certains auteurs accordent beaucoup de valeur à l'une ou l'autre des théories de la peine, d'autres non. Mais nous croyons que tous pensent que la réflexion éthique, la réflexion sur la réciprocité des droits, la modération et la réduction radicale de l'emprisonnement sont absolument nécessaires. 


\section{RÉERENCES}

ARSENAULT, R. (1989), Détention préventive et procédures judiciaires: la carrière morale du prévenu, Ottawa, Département de criminologie, Université d'Ottawa, thèse de mấtrise inédite.

BEAUMONT, G., TOCQUEVILLE, A. de (1845), «Système pénitentiaire aux États-Unis et son application en France», dans M. Perrot (ed.): Alexis de Tocqueville, Euvres complètes. Écrits sur le système pénitentiaire en France et à l'étranger, Paris, Gallimard, tome IV.

BECCARIA, C. (1764), Des délits et des peines, Paris, Flammarion, 1979.

BENTHAM, Jeremy (1791), «Panoptique; Mémoire», dans J. Bentham, Le Panoptique (précédé de «L'Gil du Pouvoir», entretien avec M. Foucault), Paris, Pierre Belfond, 1977.

BERTRAND, M.A. (1979), La Femme et le crime, Montréal, L'Aurore.

COMMISSION CANADIENNE SUR LA DÉTERMINATION DE LA PEINE (1987), Reformer la sentence. Une approche canadienne, Ottawa, Ministre des Approvisionnements et Services Canada.

DEL VECCHIO, G. (1951), Justice. An Historical and Philosophical Essay, Édimbourg, Edinburgh University Press, 1956.

DUMONT, H. , LANDREVILLE, P. (1973), «Discipline et droits des détenus dans les institutions pénales au Québec», Revue canadienne de criminologie, vol. $15, \pi^{\circ} 4$, pp. 412-434.

FOUCAULT, M. (1976), conférence publique au colloque Alternatives à la prison, Université de Montréal. Texte inédit (transcription non revue par l'auteur).

FOUCAULT, M. (1975), Surveiller et punir, Paris, Gallimard.

GaRAFALO, R. (1914), Criminology, New Jersey, Patterson-Smith.

GARAFALO, R. (1900), «Enforced Reparation as a Substitute for Imprisonment», in R. Garafalo (1914).

GARAFALO, R. (1887), Riparazione alle vittime del delitto, Irvin, Bocca.

GARLAND, D. (1986), Punishment and Welfare. A History of Penal Strategies, Hants, Gower.

GOFFMAN, E. (1961), Asiles, Paris, Les Éditions de Minuit, 1968.

HAMELIN, M. (1989), Femmes et prison, Montréal, Éditions du Méridien.

HATTEM, T. (1991), «Vivre avec ses peines: les fondements et les enjeux du contrôle et de la résistance saisis à travers l'expérience de femmes condamnées à l'emprisonnement à perpétuité», Déviance et société, vol. $15, n^{\circ} 3$, à paraître.

HATTEM, T. (1987), Condamnés à 25 ans minimum: expérience vécue et perspectives d'avenir, rapport de recherche inédit, Université de Montréal, C.I.C.C.

HATTEM, T. (1984), Le Recours d l' isolement cellulaire dans quatre établissements de détention du Québec, rapport de recherche inédit, Université de Montréal, Centre international de criminologie comparé.

HATTEM, T., PARENT, C. (1982), Les Effets négatifs d'un casier judiciaire au niveau de l'emploi, Montréal: Les Cahiers de l'École de criminologie, $\mathrm{n}^{\circ} 8$, Université de Montréal.

HATTEM, T. (1980), Prisons du Québec : prisons des pauvres, Montréal, École de criminologie, Université de Montréal, mémoire de mâ̂trise inédit. 
HULSMAN, L. H. C., BERNAT DE CELIS, J. (1982), Peines perdues. Le Système pénal en question. Paris: Le Centurion.

HULSMAN, L. H. C., (1979), «Un paradigme "criminologique” abolitionniste et la recherche sur la catégorie du crime», dans Le Fonctionnement de la justice pénale, Paris, Éditions du C.F.R.S. (orig. 1977), pp. 485-497.

JACKSON, M. (1983), Prisoners of Isolation. Solitary Confinement in Canada, Toronto, University of Toronto Press.

JACKSON, M. (1974), «Justice Behind Walls. A Study of the Disciplinary Process in a Canadian Penitentiary", Osgoode Law Journal, vol. 12, pp. 1-103.

JHERING, R. von (1877), A Evoluçaō do Direito (Zweck im Recht), Salvador, Livraria Progresso Editora, 1956 (trad. brésilienne).

LAPLANTE, J. (1989), Prison et ordre social au Québec, Ottawa, Presses de l'Université d'Ottawa.

LAURIN, Lucie (1985), Des luttes et des droits. Antécédents et histoire de la Ligue des droits de l'homme de 1936 à 1975 , Montréal, Éditions du Méridien.

LANDREVILLE, P., BLANKEVOORT, V., PIRES, A. P. (1980), Les Couts sociaux du système pénal, Montréal, École de crirninologie, Université de Montréal, rapport de recherche inédit.

LANDREVILLE, P., GAGNON, A., DESROSIERS, S. (1976a), Les prisons de par ici, Montréal, Parti-Pris.

LEMONDE, L. (1990), L'Habeas corpus en droit carcéral, Montréal, Les Éditions Yvon Blais, Inc.

LOSCHAK, D. (1984), «Mutation des droits de l'homme et mutation du droit», Revue interdisciplinaire d'études juridiques, vol. 13, pp. 49-88.

LOSCHAK, D. (1981), «Droit et non-droit dans les institutions totalitaires», dans Centre universitaire de recherches administratives et politiques de Picardie (C.U.R.A.P.P.), L'institution, Paris, P.U.F.

MATHIESEN, T. (1974), The Politics of Abolition. Essays in Political Action Theory, Londres, Martin Robertson.

MATTICK, H. W. (1973), «The Pessimistic Hypothesis and an Immodest Proposal». The Journal of the American Public Welfare Association, vol. 31, $\mathrm{n}^{\circ} 2$, pp. 2-5.

OUIMET, RAPPORT (1969), Rapport du Comité canadien de la réforme pénale et correctionnelle, Ottawa, Imprimeur de la Reine.

MOURGEON, J. (1978) Les Droits de l'homme, Paris, P.U.F.

PERROT, M. (1984), "Tocqueville méconnu», in M. Perrot (ed.), Alexis de Tocqueville, Euvres complètes, Ecrits sur le système pénitentiaire en France et à l'étranger, Paris, Gallimard, tome IV.

PIRES, A. P. (1990a), «Le Devoir de punir: le rétributivisme face aux sanctions communautaires", Revue canadienne de criminologie, vol. 32, no 3 , pp. 441-460.

PIRES, A. P. (1990b), Éthiques et réforme du droit criminel : au-delà des philosophies de la peine», communication faite au Congrès de l'ACFAS, Montreal, 1989, texte inédit.

PIRES, A. P., VALLIÈRES, S. (1987), Droits de la personne et information juridique: une recherche évaluative, Ottawa, Ministère des Approvisionnements et Services Canada.

PIRES, A. P. (1986), Critiques à la prison et principe de modération: inventaire d'extraits dans les documents canadiens, Ottawa, Étude préparée pour la Comunission canadienne sur la détermination de la peine. 
PIRES, A. P. (1983), Stigmate pénal et trajectoire sociale, Montréal, École de Criminologie, Université de Montréal, thèse de doctorat inédite.

POISSON, M. (1988), Les Femmes et le stigmate pénal, Ottawa, Département de criminologie, Université d'Ottawa, thèse de maitrise inédite.

ROCHON, G. S. (1988), La Pratique du pardon au Canada (selon la "Loi sur le casier judiciaire»), Ottawa, Département de criminologie, Université d'Ottawa, thèse de maitrise inédite.

ROTHMAN, D. J. (1981), «Doing Time: Days, Months and Years in the Criminal Justice Systems», dans H. Gross et A. von Hirsch (éd.), Sentencing, New York, Oxford University Press.

ROTHMAN, D. J. (1980), Conscience and Convenience, Boston, Little, Brown and Company.

ROTHMAN, D. J. (1975), «Behavior Modifications in Total Institutions», Hastings Center Report, vol. 5, pp. 17-24.

ROTHMAN, D. J. (1971), The Discovery of Asylum, Boston, Little, Brown and Company.

RUSCHE, G., KIRCHHEIMER, O. (1939), Punishment and Social Structure, New York, Russell and Russell, 1968.

SPENCER, H. (1860), «Prison-Ethics», dans H. Spencer (1966), Essays : Scientific, Political and Speculative, Osnabrück, Otto Zeller, vol. III.

VILLEY, M. (1979), «La doctrine du droit dans l'histoire de la science juridique», préface à E. Kant, Métaphysique des mocurs (Première partie: Doctrine du droit), Paris, J. Vrin, pp. 7-26.

VON HIRSCH, A. (1976), Doing Justice, The Choice of Punishments. Report of the Committee for the Study of Incarceration, Boston, Northeastern University Press. 